\title{
Prevalence of Equinus in Patients Diagnosed with Plantar Fasciitis
}

\author{
by EM Wenzel, MT(ASCP), DPM ${ }^{1}$, Z Kajgana , DPM ${ }^{1}$, KD Kelley, DPM ${ }^{2} \rrbracket$, KM Mason DPM, C Ped ${ }^{3}$, \\ JS Wrobel, DPM, MS ${ }^{4}$, DG Armstrong DPM, PhD ${ }^{5,6}$
}

The Foot and Ankle Online Journal 2 (3): 1

Background: Plantar fasciitis is one of the most common complaints seen in podiatric practice. Typically, the diagnosis is made based on clinical presentation and patient history. Biomechanics is believed to also contribute to the onset of this condition through a decreased ankle joint range of motion.

Methods: The design was a retrospective case-control study of patients with plantar fasciitis $(n=23)$ and a control group $(n=54)$. Medical records from the Scholl Foot Clinic at Rosalind Franklin University were abstracted for measurements for ankle joint dorsiflexion with the knee extended and flexed along with other patient variables.

Results: Patients diagnosed with plantar fasciitis did not have a significant decrease in ankle joint dorsiflexion $(p=0.8979)$. A significantly higher body mass index (BMI) was noted in patients diagnosed with plantar fasciitis as compared to the control group (34+/- 7.99 v. $29+/-5.81 ; p=0.0046)$. Increased plantar fasciitis was also noted in cavus foot structure $(p=0.0323)$ and in women $(p=0.0147$ for left and $p=0.0250$ for right). Conclusions: An increased BMI, cavus foot structure, and female gender were found to be associated with a diagnosis of plantar fasciitis.

Key words: Plantar fasciitis, cavus foot, BMI.
Accepted: February, 2009
Published: March, 2009

This is an Open Access article distributed under the terms of the Creative Commons Attribution License. It permits unrestricted use, distribution, and reproduction in any medium, provided the original work is properly cited. @The Foot and Ankle Online Journal (www.faoj.org)

$\mathrm{P}$ revious research has estimated that approximately two million Americans are annually affected by plantar fasciitis ${ }^{1}$ and it is further estimated that up to $25 \%$ of all foot injuries are due to plantar fasciitis. ${ }^{2}$

\footnotetext{
Address correspondence to: KD Kelly, DPM PGY-2 resident in Podiatric Medicine and Surgery at the Central Alabama Veterans Health Care System in Montgomery, Alabama.

E-mail: Kristin.Kelley@va.gov

${ }^{1}$ PGY-II Podiatry Resident, Forum Health/Western Reserve Care System Podiatric Residency Program

${ }^{2}$ PGY-II Chief Podiatry Resident, Central Alabama Veterans' Health Care System

${ }^{3}$ Chair, Department of Biomechanics and Orthopedics

${ }^{4}$ Director, Outcomes Research, Center for Lower Extremity and Ambulatory, CLEAR

${ }^{5}$ Chair, Research; Assistant Dean; Professor of Surgery

${ }^{6}$ Director, Center for Lower Extremity and Ambulatory Research, CLEAR
}

Plantar fasciitis, the inflammation of the plantar aponeurosis (most often of the central portion), is commonly responsible for the symptoms of the heel, midfoot and forefoot pain. ${ }^{1,3,4}$ Its diagnosis is usually made solely on history and physical examination despite the etiology of being poorly understood. ${ }^{5,6}$

It has been hypothesized that anatomical, biomechanical and environmental factors may influence the inflammation of the plantar aponeurosis. ${ }^{7}$ Anatomical factors include weight, tarsal coalition, and fat atrophy, where as biomechanical and environmental factors include tight Achilles tendon and poor footwear or walking barefoot respectively. ${ }^{8}$ 


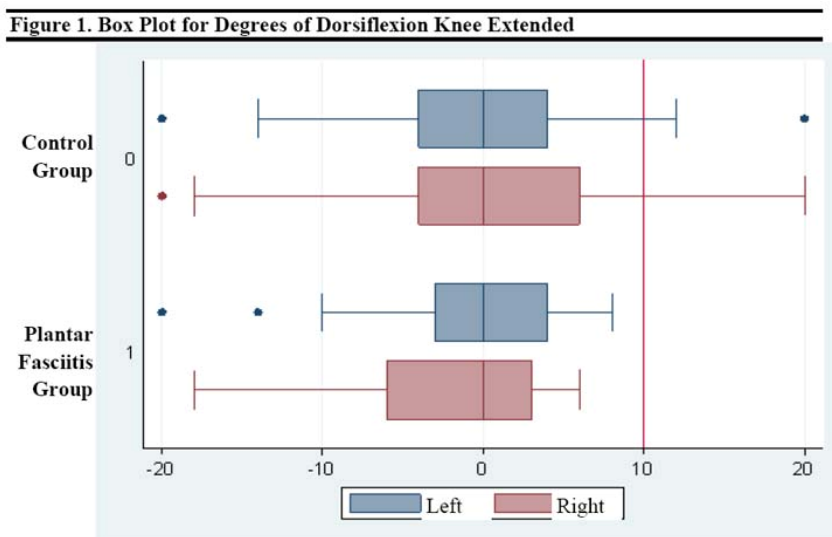

Figure 1 Box Plot for Degrees of Dorsiflexion Knee Extended.

Furthermore, a plantarflexed ankle joint allows for the contraction of the fascial tissue which is stretched during the midstance possibly causing microtears which induce a reparative inflammatory response. ${ }^{6}$ This latter consideration has led us to believe that the chief etiology might indeed be of a biomechanical nature (i.e. tight Achilles tendon), since a majority of runners have a tight Achilles tendon which leads to the plantarflexed ankle. ${ }^{6}$

Values for dorsiflexion of the ankle joint vary in the literature significantly and range from 0 degrees to 25 degrees. ${ }^{9}$ However, for the purpose of this study it was necessary to classify the minimum amount of motion that must occur at the ankle joint for a patient to be classified as having normal ankle joint range of motion and those with less being classified as having an equinus. While it has been found that static measures and dynamic measures are not well correlated, the most widely accepted values in the literature for static measurements, and for the purpose of this study, state that the minimum amount of dorsiflexion necessary at the ankle for the normal gait is 10 degrees of motion. . $^{10,11,12,13,14,15}$

We hypothesized that patients complaining of plantar fasciitis would have an increased incidence of equinus deformity as compared to a control group of patients who do not complain of symptoms relating to plantar fasciitis and who had no previous history of a plantar fasciitis diagnosis.

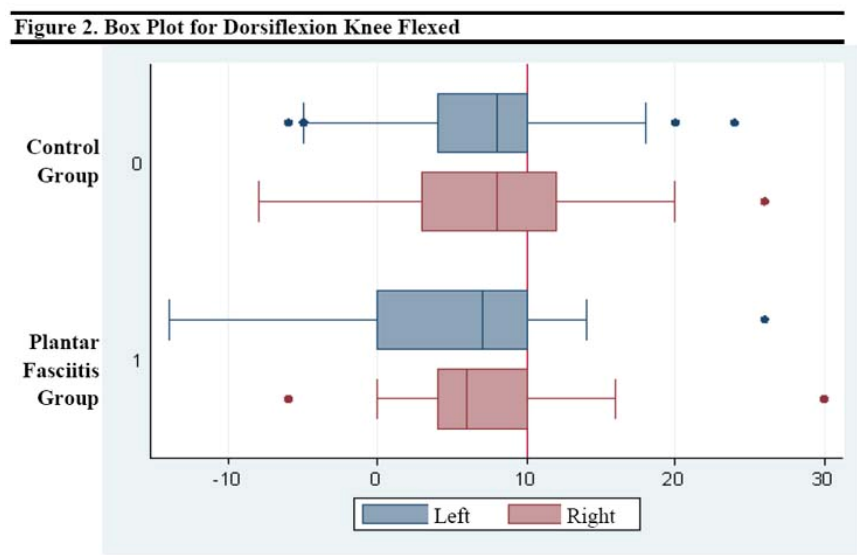

Figure 2 Box Plot for Dorsiflexion Knee Flexed.

\section{Methods}

A retrospective study of patient records was performed at the Rosalind Franklin Student Clinics. The study protocol and procedures were approved by the University's Institutional Review Board. Consecutive records from the archives were pulled from podiatric patients with a diagnosis code of plantar fasciitis, beginning before and up to March 3rd, 2005 resulting in 48 patient records. These were selected for review based upon complete record of the following criteria: age, height, weight, occupation, typical shoe gear, what elicited pain for the patient, whether radiographs were obtained and the findings, bilateral ankle joint range of motion (knee extended and flexed) and foot structure. Any chart with incomplete or missing data according to the criteria previously set forth was excluded resulting in a patient population of 23 (17 women and 6 men) with a mean \pm standard deviation (SD) age of $49 \pm 11$ years (range 25 to 69 years).

A control group was formed from consecutive patient charts without a diagnosis code of plantar fasciitis or history of ankle joint trauma. These charts were reviewed and included upon complete notation of the following criteria: age, height, weight, occupation, typical shoe gear, bilateral ankle joint range of motion (knee extended and flexed) and foot structure. 


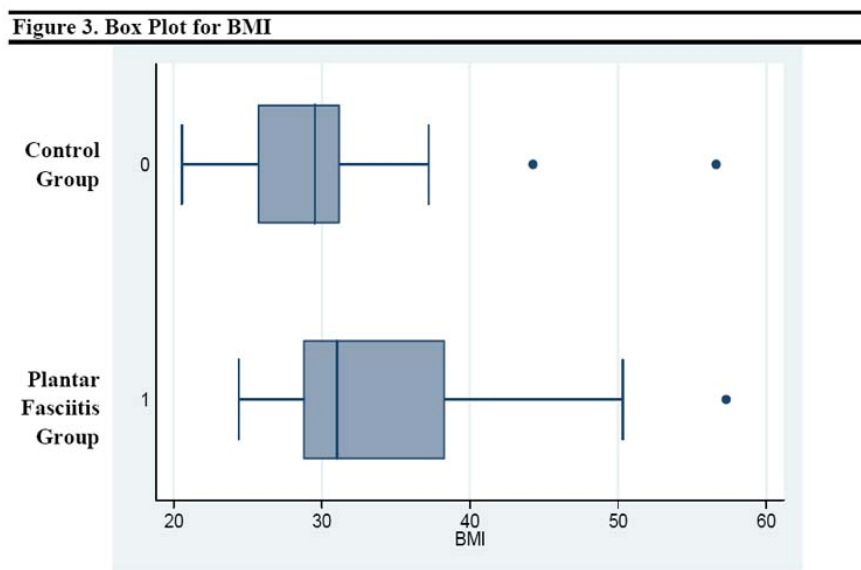

Figure 3 Box Plot for BMI.

Following chart review and exclusion criteria a group of 54 patients was formed to serve as the control group with a mean age of $49 \pm 11$ years (range 18 to 68 years).

Data analysis was performed using STATA (version 9.1, College Station, TX) to determine whether a significant difference existed in the prevalence of equinus between the plantar fasciitis group and the control group. Other variables were also analyzed to determine the interrelationship with plantar fasciitis including BMI, gender, shoe gear, foot structure, and occupation. For continuous predictor variables oneway analysis of variance (ANOVA) with Bartlett's test for equal variance was used. For categorical predictor variables ANOVA with Scheffe's Post Hoc test was utilized. For dichotomous predictor variables Fisher's exact test was used.

\section{Results}

While a trend towards plantar fasciitis patients having decreased ankle dorsiflexion was expected, these findings were not significant $(\mathrm{p}=0.8979)$ (Figs. 1 and 2). Mean dorsiflexion $\pm \mathrm{SD}$ of the left ankle was found to be $-1.91 \pm 7.44$ with the knee extended and $5.96 \pm 7.85$ with the knee flexed. For the right ankle these values were $-1.74 \pm 6.12$ knee extended and $6.57 \pm 7.19$ knee flexed (Table 1).
A significant difference was found to exist between the control group and the plantar fasciitis group in BMI ( $p=0.0046)$; those being diagnosed with plantar fasciitis having an increase in BMI. (Fig. 3) A similar trend in respect to weight $(\mathrm{p}=0.0394)$ was also noted. An increased incidence of plantar fasciitis was found in those patients with a cavus foot structure ( $p$ $=0.0323)$. An increase in plantar fasciitis was found in women $(p=0.0147$ for left and $p=0.0250$ for right) (Table 2). No correlation between the patients with plantar fasciitis and their occupation, shoe gear or age was found.

\section{Discussion}

In this retrospective study patients with equinus were in fact not found to have a significant difference in the formation of plantar fasciitis $(\mathrm{p}=0.8979)$ (Figures $1,2)$. A trend was noted in increased gastroc-soleal $(32 \%$ greater $)$ and gastrocnemius equinus $(20 \%$ increase) as compared to the control group. Despite these trends a statistical significance was not found to exist disproving our initial hypothesis.

The etiology of equinus deformity has spawned much research into determining its associated causes and complications. Some that have been more anecdotal include increased weight, age, foot structure, sex, occupation, or poor shoe gear. Another theory has been that if an ankle joint that is already compromised by an abnormally limited range of motion, as in equinus formation, is then subjected to unusual stress or exertion, such as repetitive daily trauma to the surrounding structures, conditions such as plantar fasciitis are a probable result. ${ }^{6}$

Although its link to plantar fasciitis may seem obvious, there are few articles that directly state or have adequate statistical value to prove the link. Riddle, et al., state that equinus, BMI, and work related weightbearing are the most relevant risk factors in predicting plantar fasciitis. ${ }^{1}$ However, in their more recent article on the same topic they state BMI as being the only contributory variable for a reduced Lower Extremity Functional Score. ${ }^{16}$ 
Table 1. Characteristics of Plantar Fasciitis Patients and Control Group Patients

Plantar Fasciitis Group

$(\mathrm{N}=23)$

\section{Control Group}

(N=54)
Variable

Age (years)

Height (inches)

Weight (pounds)

BMI (pounds/height` $`$ )

Left Ankle Dorsiflexion (degrees)

Knee Extended

Knee Flexed
Mean \pm Standard Deviation

$48.70 \pm 10.66$

$63.96 \pm 3.43$

$199.61 \pm 51.78$

$34.20 \pm 7.99$

$-1.91 \pm 7.44$

$5.96 \pm 7.85$
Mean \pm Standard Deviation

$49.04 \pm 10.55$

$65.5 \pm 4.24$

$178.70 \pm 33.26$

$29.41 \pm 5.81$

$0.39 \pm 7.11$

$7.20 \pm 6.29$

\section{Right Ankle Dorsiflexion (degrees)}

Knee Extended

$-1.74 \pm 6.12$

$0.09 \pm 7.71$

Knee Flexed

Other authors such as Warren found a higher incidence of equinus in those patients who had not had symptoms of plantar fasciitis. ${ }^{17}$

As obesity is an often cited cause of plantar fasciitis the weight and BMI calculations of the plantar fasciitis group were compared to the control group. ${ }^{1}$ This was performed using the Centers for Disease Control and Prevention (CDC) guidelines for calculation and categorization: normal (BMI <25), overweight $(25-<32)$, obese $(32-<36)$, grossly obese $(36-<40)$, and morbidly obese (greater than 40) based on the patient's height and weight. ${ }^{18}$ Data analysis revealed a significant difference existed between the two groups $(p=0.0046)$ for BMI and also for weight $(p=0.0394)$ supporting the theory that BMI and weight are predictive for the development of plantar fasciitis. (Fig. 3)

Gender has long been thought to play a role in the development of plantar fasciitis and women are considered to be the group most often affected with plantar fasciitis. ${ }^{6}$
Although this can be attributed to shoe gear difference when compared to the control group, a trend that did not reach significance could be seen in a larger number of women being afflicted with plantar fasciitis than the more evenly distributed gender of the control group ( $74 \%$ compared to $59 \%$ ). This study revealed that women had a significant decrease in ankle joint dorsiflexion with the knee extended ( $p$ $=0.0147$ for left and $\mathrm{p}=0.0250$ for right). The shoe gear of men versus women was analyzed and no significant finding could be noted. This supports the theory that decreased ankle joint dorsiflexion might be another contributory factor in plantar fasciitis development in women.

Foot structure is commonly noted a predictive factor in the formation of plantar fasciitis, pes planus being more susceptible to its formation. ${ }^{7,8}$ In this study it was found that pes cavus feet actually had an increased incidence of plantar fasciitis than pes planus feet $(p=0.0323)$. Other factors such as age, occupation, and shoe gear were analyzed and no significant finding could be noted in the formation of plantar fasciitis. 


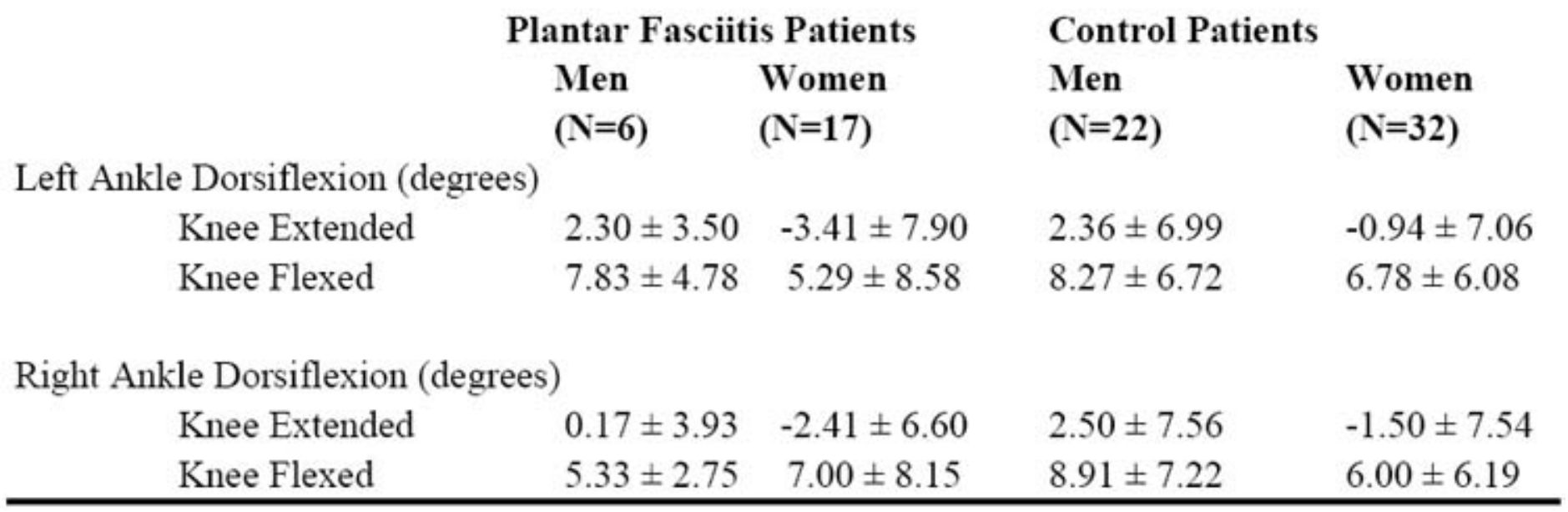

There are several primary limitations for this study. First, since research was collected from previous medical chart data that was documented by students, there is a potential for evaluator variability. It is safe to assume that the ankle joint ranges of motion measurements for each patient were not done by the same person and there is a potential for differing amounts of force applied during dorsiflexion exam. ${ }^{8}$ Since the students taking the measurements were supervised by experienced clinicians, any gross errors or miscalculations are less likely. Furthermore, Martin and McPoil state that in ankle dorsiflexion measurements, interrater reliability can be expected. ${ }^{19}$

Because patients self report their height and weight there is a potential under or overestimation of the BMI. Recent research however has shown that underestimation is more likely than overestimation in self reported groups. ${ }^{20}$ This study was also limited by lack of consistent detail provided by the medical charts this study required, therefore resulting in a reduction in sample size from the initial group of patients diagnosed with plantar fasciitis. However, a larger sample size may have provided the power to detect differences in ankle joint dorsiflexion. A limitation related to the data analysis is that this study used the foot as a unit and rather than using a clustered analysis of feet.

\section{Conclusion}

The authors hypothesized that a greater incidence of equinus deformity could be found among patients with plantar fasciitis than in those without history of plantar fasciitis. Upon examination of the data it was found that this initial hypothesis could not be supported by the analysis. Females diagnosed with plantar fasciitis were found to have to have a significant decrease in ankle joint dorsiflexion with the knee extended. An increased BMI and weight as well as a cavus foot structure were the only variables that showed a significant increase among all patients diagnosed with plantar fasciitis as compared to the control group. Other variables such as occupation, age, and shoe gear were not found to be of statistical significance when compared to a control group.

The results of this study suggest that weight loss should be utilized as a primary conservative treatment for patients diagnosed with plantar fasciitis who have a higher than normal BMI and weight before surgical alternatives are employed. Further prospective study with a larger patient population is warranted ensuring consistent charting and including clinically measured height and weight data. 


\section{References}

1. Riddle DL, Pulisic M, Pidcoe P, Johnson RE: Risk factors for plantar fasciitis: A matched case-control study. J Bone Joint Surg 85A: $872-877,2003$.

2. Landorf KB, Keenan AM, Herbert RD: Effectiveness of different types of foot orthoses for the treatment of plantar fasciitis. JAPMA 94 (6): 542 - 549, 2004.

3. Ward ED, Smith KM, Cocheba JR, Patterson PE, Phillips RD: In vivo forces in the plantar fascia during the stance phase of gait. JAPMA 93 (6): 429 - 442, 2003.

4. DiGiovanni CW, Kuo R, Tejwani N, Price R, Hansen ST Jr, Cziernecki J, Sangeorzan BJ: Isolated gastrocnemius tightness. J Bone Joint Surg 84A: 962 - 970, 2002.

5. Gheluwe BV, Kirby KA, Roosen P, Phillips RD: Reliability and accuracy of biomechanical measurements of the lower extremities. JAPMA 92 (6): 317 - 326, 2002. 6. Singh D, Angel J, Bentley G, Trevino SG: Fortnightly review: Plantar fasciitis. BMJ 315 (July): 172 - 175, 1997.

7. Richie DH: The best treatments for plantar heel pain. Podiatry Management 135, August 2002. Access date and url is required.

8. Martin JE, Hosch JC, Goforth WP, Murff RT, Lynch DM, Odom RD: Mechanical treatment of plantar fasciitis: A prospective study. JAPMA 91 (2): 55 - 62, 2001.

9. Saxena A, Kim W: Ankle dorsiflexion in adolescent athletes. JAPMA 93 (4): 312 - 314, 2003.

10. Knutzen KM, Price A. Lower extremity static and dynamic relationships with rearfoot motion in gait. JAPMA 84 (4): 171 $180,1994$.
11. Nuber GW. Biomechanics of the foot and ankle during gait. Clin Sports Med 7 (1): 1 - 13, 1988.

12. Lavery LA, Armstrong DA, Boulton AJM. Ankle equinus deformity and its relationship to high plantar pressure in a large population with diabetes mellitus. JAPMA 92 (9): 479 - 482, 2002.

13. Wrobel JS, Connolly JE, Beach ML: Associations between static and functional measures of joint function in the foot and ankle. JAPMA 94 (6): 535 - 541, 2004.

14. Winter DA. Kinematic and kinetic patterns in human gait: variability and compensating effects. Human Mov Sci 3: 51 $76,1984$.

15. Lamm BM, Paley D, Herzenberg JE: Gastrocnemius soleus recession: A simpler, more limited approach. JAPMA 95 (1): 18 $-25,2005$.

16. Riddle DL, Pulisic M, Sparrow K: Impact of demographic and impairment-related variables on disability associated with plantar fasciitis. Foot Ankle Int 5: 311 - 317, 2004.

17. Warren BL: Plantar fasciitis in runners: Treatment and prevention. Sports Med 10 (5): 338 - 345, 1990.

18. Bender R, Joekel KH, Trautner C, Spraul M, Berger M: Effect of age on excess mortality in obesity. JAMA 281: 1498 1504, 1999.

19. Martin RL, McPoil TG: Reliability of ankle goniometer measurements: A literature review. JAPMA 95 (6): 564 - 572, 2005.

20. Kuczmarski MF, Kuczmarski, RJ, Najjar M: Effects of age on validity of self-reported height, weight, and body mass index: Findings from the third national health and nutrition examination survey. J Am Diet Assoc. 101 (1): 28 - 34, 2001. 